\title{
Recent advances in computational biology, bioinformatics, medicine, and healthcare by modern OR
}

\author{
Gerhard-Wilhelm Weber • Jacek Blazewicz • \\ Marion Rauner • Metin Türkay
}

At the occasion of the 25th European Conference on Operational Research, EURO $X X V$ 2012, July 8-11, 2012, in Vilnius, Lithuania (http://www.euro-2012.1t/), the Central European Journal of Operations Research (CJOR; http://www.springer.com/ business+\%26+management/operations+research/journal/10100) invited to submissions of papers to a special issue on Recent Advances in Computational Biology, Bioinformatics, Medicine and Healthcare by Modern OR. EURO 2012 was organised by the Lithuanian Operational Research Society and the University of Vilnius, together with The Association of European Operational Research Societies (EURO; http:// www.euro-online.org/). The conference participants were researchers, academicians, and practitioners interested in Operational Research $(O R)$, mathematical modelling and quantitative economic analysis. There were more than 2,000 participants who came from various countries of the world, making the Vilnius conference to one the most successful EURO conferences ever.

\section{G.-W. Weber $(\bowtie)$}

Institute of Applied Mathematics, Middle East Technical University, 06531 Ankara, Turkey

e-mail: gweber@metu.edu.tr

\section{J. Blazewicz}

Institute of Computing Science, Poznan University of Technology, ul. Piotrowo 2, 60-965 Poznan, Poland

e-mail: jblazewicz@cs.put.poznan.pl

\section{Rauner}

Department Innovation and Technology Management, University of Vienna, Bruenner Str. 72, 1210 Vienna, Austria

e-mail: marion.rauner@univie.ac.at

\section{Türkay}

Department of Industrial Engineering, Koc University, Rumelifeneri Yolu, Sariyer, 34450 Istanbul, Turkey e-mail: mturkay@ku.edu.tr 
Among the relatively new, but emerging fields of $O R$, there are both Healthcare and, closely related, Computational Biology, Bioinformatics and Medicine. That $O R$ could get established in the sectors of biology, health and medicine and, through its quantitative and interdisciplinary instruments, enriches their methodologies and contributes to and their real-world applications, has been a great achievement. Those applications are of such a great importance for life quality and life expectation of the people on earth. All four fields have a direct and natural relation to the sciences of biology and mathematics, but also to informatics and data mining. Healthcare traditionally puts a strong emphasis on management and, in particular, organization, too.

With our special issue in CJOR, we hope to support the collaboration in the area of these fields and to represent state-of-the-art contributions presented and discussed at the occasion of EURO 2012. It should further encourage excellent research and interaction in these vibrant fields of modern $O R$ and among them, and to stimulate excellent future science hosted and fostered in our $O R$ family.

The importance of OR applications in biology, bioinformatics, medicine and healthcare is highly reflected by the growing number of contributions to international conferences and scientific journals in the last years. Both the EURO Working Group "OR Applied to Health Care" (ORAHS; http://orahs.di.unito.it/) and EURO Working Group on "Operational Research in Computational Biology, Bioinformatics and Medicine” (EURO-CBBM; http://euro-cbbm.ku.edu.tr/) observe an increasing number of participants in their streams at EURO conferences and in their further scientific events of all formats. This growth has become reflecting itself in an increasing number of publications and in special issues prepared by members of both working groups, respectively. One special issue on healthcare appeared in CJOR in 2005, while another one on energy, health, and life sciences was published in CJOR in 2009. Please find some further special issues of CJOR listed below. Now, at this time, we-members and representatives of the two groups-are going together with our joint special issue.

Subsequently, we will shortly summarize the articles of this special issue that remained after rigorous reviewing processes.

The contribution of Dias et al. (2013), proposed a genetic algorithm for beam angle optimization to find the best set of angles that should be used in Intensity Modulated Radiotherapy Treatment (IMRT) planning for advanced cancer treatment. A neural network was used as a surrogate model to calculate the fitness of most of the individual patients in the population. They illustrated that this genetic algorithm could provide improved solutions compared to the usual equidistant solution applied in clinical practice.

Comparison of molecular structures is an important step in solving various problems derived from computational biology. The paper of Szachniuk et al. (2013), presents MCQ4Structures, a new tool for structural similarity computation based on molecule tertiary structure representation in torsional angle space. The authors discuss its unique features as compared with the other measures, and they demonstrate its experimental use in comparison of a number of 3D structures as well as evaluation of predictions within RNA-Puzzles contest. 
The objectives of the paper by Lai et al. (2013), are three folds. Firstly, the primary objective is to use one single automatic method (post-initialisation) to reproduce the six classes for the 663 patients and to classify the remaining 413 patients. Secondly to achieve this, they used semi-supervised fuzzy c-means with various distance metrics and initialisation techniques. Thirdly, the clinical characteristics of the 413 patients are examined by comparing with the 663 patients. Their experiments use various amount of labelled data and 10-fold cross validation to reproduce and evaluate the classification. Examination of clinical characteristics indicates significant associations between classification and clinical parameters. More importantly, association between classification and survival based on the survival curves is shown.

Le Thi et al. (2013), propose a new optimization approach based on Difference of Convex functions (DC) programming and DC Algorithm (DCA) for the multiple sequence alignment in its equivalent binary linear program, called "Maximum Weight Trace" problem. The algorithm converges after finitely many iterations to a binary solution while it works in a continuous domain. First numerical experiments on benchmark data demonstrate the efficiency of their proposed algorithm DCAMSA, which outperforms some standard methods.

In their work, Marinakos et al. (2013), design a decisions support solution for Greek pharmacies comprising a cash flow management system for early warning of financial distress and a financial advisor. They employ a neural network, and a linear regression module to forecast future cash reserves based on past profitability ratios. Their model aims to inform and consult various members of the pharmaceutical chain and, thus, to reduce its financial risk.

Üney-Yüksektepe (2013) presents a novel extension of cutting decision tree (CDT) approach for binary classification by incorporating the second goal of maximizing the distance of the correctly classified instances to the misclassification region. The effect of the secondary objective is illustrated on a number of well-known bioinformatics problems including the Wisconsin Breast Cancer database, Buba Liver Disorders, Blood Transfusion and Pima Indian Diabetes.

Swiercz et al. (2013) introduces a new approach to applying hyper-heuristic algorithms to solve combinatorial problems. The authors propose a unified encoding of a solution and a set of low-level heuristics that are domain-independent. This new approach is shown to be very effective in generating a set of approximate solutions on DNA sequencing by hybridization problems that contains errors.

Acknowledgments We are very grateful to Editor in Chief of CJOR, Prof. Ulrike Leopold-Wildburger from Karl-Franzens-University, Graz, Austria, for her interest, confidence and continuous support at all stages of the preparation of this special issue. Furthermore, we extend many cordially thanks to Mr. Dilip Rajasekar and Mrs. Sujitha Magdalene Sunder from Springer Verlag for their excellent guidance through all the review and communication processes related with the submissions. We guest editors wish all readers an enjoyable, insightful and inspiring lecture of the contributions of this CJOR special issue! In fact, we sincerely hope that our special issue will reveal the Central European Journal of Operations Research as a Premium journal of Science which strongly supports Operational Research in Central Europe, in overall Europe and in all over the word. 


\section{References}

Dias J, Rocha H, Ferreira B, do Carmo Lopes M (2013) A genetic algorithm with neural network fitness function evaluation for IMRT beam angle optimization. Central Eur J Oper Res. doi:10.1007/ s10100-013-0289-4

Lai DTC, Garibaldi JM, Soria D, Roadknight CM (20313) A methodology for automatic classification of breast cancer immunohistochemical data using semi-supervised Fuzzy c-Means. Central Eur J Oper Res. doi:10.1007/s10100-013-0318-3

Le Thi HA, Pham Dinh T, Tayeb Belghiti M (2013) DCA based algorithms for multiple sequence alignment (MSA). Central Eur J Oper Res. doi:10.1007/s10100-013-0324-5

Marinakos G, Daskalaki S, Ntrinias T (2013) Defensive financial decisions support for retailers in Greek pharmaceutical industry. Central Eur J Oper Res. doi:10.1007/s10100-013-0325-4

Swiercz A, Burke EK, Cichenski M, Pawlak G, Petrovic S, Zurkowski T, Blazewicz J (2013) Unified encoding for hyper-heuristics with application to bioinformatics. Central Eur J Oper Res. doi:10.1007/ s10100-013-0321-8

Szachniuk M, Zok T, Popenda M (2013) MCQ4Structures to compute similarity of molecule structures. Central Eur J Oper Res doi:10.1007/s10100-013-0296-5

Üney-Yüksektepe F (2013) A novel approach to cutting decision trees. Central Eur J Oper Res. doi:10. 1007/s10100-013-0312-9

\section{Additional References}

Central European Journal of Operations Research (2005) Special issue on "Quantitative Decision Support for Health Services”. In: Rauner MS, Behrens D, Wild C (eds) Central European Journal of Operations Research, vol 13, no 4

Central European Journal of Operations Research (2007) Special issue on "OR for Better Management of Sustainable Development" at the occasion of EURO XXI 2006. In: Pick1 SW, Weber G-W, Zachariasen M (eds) Central European Journal of Operations Research, vol 15, no 3

Central European Journal of Operations Research (2008) Special issue at the occasion of 5th EUROPT workshop "Advances in Continuous Optimization" (Reykjavik, Iceland, June 30-July 1, 2006). In: Stein O, Still G, Terlaky T, Weber G-W (eds) Central European Journal of Operations Research, vol 16, no 2

Central European Journal of Operations Research (2011) “OR and Environmental Management", Feature Sample at the Occasion of EURO 2009. In: Letmathe P, Tuma A, Weber G-W (eds) Central European Journal of Operations Research, vol 19, no 4

Central European Journal of Operations Research (2012) Special issue on OR in the financial sector at the occasion of the 24th European conference on operational research, EURO XXIV, July 11-14, 2010, Lisbon, Portugal, feature sample, In: Kropat E, Weber G-W (eds) Feature sample, integrated into Central European Journal of Operations Research, vol 20, no 3, pp 485-557 